\title{
The circadian syndrome predicts cardiovascular disease better than metabolic syndrome in Chinese adults
}

\author{
Z. Shi ${ }^{1}$ (D) J. Tuomilehto ${ }^{2}$, N. Kronfeld-Schor ${ }^{3}$ (D, K. G. Alberti ${ }^{4}$, N. Stern ${ }^{5}$, A. El-Osta ${ }^{6,7}$ (D, C. Bilu ${ }^{3}$, H. Einat ${ }^{8}$ \& \\ P. Zimmet ${ }^{5,9}$ \\ From the ${ }^{1}$ Human Nutrition Department, College of Health Sciences, QU Health, Qatar University, Doha, Qatar; ${ }^{2}$ Department of Public Health, \\ University of Helsinki, Helsinki, Finland; ${ }^{3}$ School of Zoology, Tel Aviv University, Tel Aviv, Israel; ${ }^{4}$ Department of Endocrinology and \\ Metabolism Imperial, College London, London, UK; ${ }^{5}$ Sagol Epigenetics Center, Tel Aviv Medical Center, Tel Aviv, Israel; ${ }^{6}$ Department of \\ Diabetes, Central Clinical School, Monash University, Melbourne, Vic., Australia; ${ }^{7}$ The Chinese University of Hong Kong, Hong Kong SAR, \\ China: ${ }^{8}$ School of Behavioral Sciences, Tel Aviv-Yaffo Academic College, Tel Aviv, Israel; and ${ }^{9}$ Department of Diabetes, Central Clinical \\ School, Monash University, Melbourne, Vic., Australia
}

Abstract. Shi Z, Tuomilehto J, Kronfeld-Schor N, Alberti KG, Stern N, El-Osta A, Bilu C, Einat H, Zimmet P (QU Health, Qatar University, Doha, Qatar; University of Helsinki, Helsinki, Finland; Tel Aviv University, Tel Aviv, Israel; College London, London, UK; Tel Aviv Medical Center, Tel Aviv, Israel; Monash University, Melbourne, Vic., Australia; The Chinese University of Hong Kong, Hong Kong SAR, China; Tel Aviv-Yaffo Academic College, Tel Aviv, Israel; Monash University, Melbourne, Vic., Australia). The circadian syndrome predicts cardiovascular disease better than metabolic syndrome in Chinese adults. $J$. Intern. Med., 2020;

Background. To compare the predictive value of the circadian syndrome (CircS) and Metabolic syndrome (MetS) for cardiovascular disease.

Method. We used the data of 9360 Chinese adults aged $\geq 40$ years from the 2011 China Health and Retirement Longitudinal Study (CHARLS). Of the participants, 8253 people were followed in the 2015 survey. MetS was defined using the harmonized criteria. CircS was based on the components of the International Diabetes Federation (IDF) MetS plus short sleep and depression. The cut-off for CircS was set as $\geq 4$. Multivariable logistic regression analysis was used to examine the associations.

Results. The prevalence of CircS and MetS was 39.0\% and $44.7 \%$. Both MetS and CircS were directly associated with prevalent CVD. The odds ratios for prevalent CVD comparing CircS with MetS, respectively, were $2.83(95 \% \mathrm{CI} 2.33-3.43)$ and 2.34 (1.93-2.83) in men, and 2.33 (1.98-2.73) and 1.79 (1.53-2.10) in women. Similar associations were found for incident CVD. The five-year incidence of CVD was $15.1 \%$ in CircS and $14.0 \%$ in MetS. The number of CircS components has a better predictive power for both prevalent and incident CVD than those of Mets components as indicated by the area under the ROC (AUC). AUC values for CVD in 2011 were higher for CircS than MetS in both men $(0.659$ (95\%CI $0.634-0.684)$ vs 0.635 (95\%CI 0.610-0.661)) and women (0.652 $(95 \%$ CI $0.632-0.672)$ vs 0.619 (95\%CI $0.599-$ $0.640))$.

Conclusion. The circadian syndrome is a strong and better predictor for CVD than the metabolic syndrome in Chinese adults.

Keywords: adults, cardiovascular disease, Chinese, circadian syndrome, metabolic syndrome.

\section{Introduction}

Cardiovascular disease is a major threat to human health globally [1]. Identifying the risk factors for CVD is vital for its prevention. A variety of factors have been found to be associated with CVD in China, mostly related to sedentary lifestyles and high consumption of an unhealthy diet [2].
Metabolic syndrome (MetS) is defined by a cluster of risk factors for cardiovascular disease and includes raised blood pressure, dyslipidaemia (raised triglycerides and lowed high-density lipoprotein cholesterol), raised fasting glucose and central obesity [3]. It is well recognized that MetS is associated with an increased risk of CVDs and the prevalence of MetS is increasing worldwide [4, 5]. In China, the prevalence of MetS was $33.9 \%$ in 
2010 [6]. However, there is debate about the underline mechanism of MetS and suggested mechanisms include insulin resistance [7], central obesity-related inflammation [8] and genetics [9].

Recently, circadian dysfunction has been proposed as an important underlying aetiological factor for the metabolic syndrome [10]. The concept of CircS is built on the fact that several chronic disorders including obesity, hypertension, dyslipidaemia, type 2 diabetes, depression, sleep disorder and nonalcoholic fatty liver disease (NAFLD) have a strong link with circadian rhythms. Modern lifestyle (e.g. sleep deprivation, high-energy diet and shift work) and the use of artificial light can cause circadian rhythm disruption, with different health outcomes [10]. Disturbances in sleep patterns and depressive symptoms have a negative effect on the circadian rhythm of numerous body functions that increase the risk of CVD. Therefore, it has been suggested that the circadian syndrome (CircS) should be considered as a novel CVD risk cluster [10]. Despite the concept of CircS, no definition of CircS has been proposed so far.

There is no study yet that has examined the association between the circadian syndrome and cardiovascular disease so it is not known whether it is a better predictor for CVD than the metabolic syndrome. The aim of this study was to answer the question using data from China Health and Retirement Longitudinal Study (CHARLS).

\section{Method}

Data source and analytical sample

CHARLS is a prospective study of adults aged $\geq 45$ years conducted in 28 provinces in China, but some people aged 40-44 years attended the baseline examination [11]. Ethical approval for the CHARLS study was reviewed by the Peking University institutional review board (IRB). Informed consent was obtained from all the participants prior to their inclusion in the study. In the current study, the 2011 baseline and 2015 followup data were used.

At baseline, a total of 17708 participants completed a face-to-face interview. Height, weight, waist circumference and blood pressure were measured by trained health workers. Fasting blood samples were measured for glucose and lipids. The response rate was $80.5 \%$. All the baseline participants were invited to participate in the follow-up survey in 2015. In the current analysis, we used the data of 9360 Chinese adults (4258 men and 5102 women) aged $\geq 40$ years from the 2011 CHARLS. Of the participants, 8253 participants were followed in the 2015 survey (Fig. 1).

\section{Exposure measures: Metabolic syndrome and circadian syndrome}

Fasting blood samples were collected and stored at $-70^{\circ} \mathrm{C}$ at the Chinese Center for Disease Control and Prevention. Biomarkers including high-sensitivity C-reactive protein (CRP), total high-density lipoprotein (HDL) and low-density lipoprotein (LDL) cholesterols and triglycerides, glycated haemoglobin (HbA1c), fasting plasma glucose and haemoglobin were measured at the Younanmen Center for Clinical Laboratory of Capital Medical University [12]. Quality control samples were used daily during the testing period, and results were within the target range (two standard deviations of mean control concentrations).

Blood pressure was measured, whilst seated, by trained nurses using HEM-7200 electronic monitor (Omron, Dalian). The mean of three measurements was used. Hypertension was defined as systolic blood pressure $\geq 140 \mathrm{mmHg}$ and/or diastolic blood pressure $\geq 90 \mathrm{mmHg}$ or use of antihypertensive medications.

Depressive symptoms were assessed using the tenquestion version of the Center for Epidemiologic Studies-Depression scale (CES-D). Participants with a CES-D score of $\geq 10$ were defined as having depressive symptoms [13].

Metabolic syndrome was defined according to the definition in 'Harmonizing the metabolic syndrome: a joint interim statement of the International Diabetes Federation Task Force on Epidemiology and Prevention; National Heart, Lung and Blood Institute; American Heart Association; World Heart Federation; International Atherosclerosis Society; and International Association for the Study of Obesity' [3] (Table 1). Having $\geq 3$ of the following components was defined as having MetS: elevated waist circumference $(\geq 85 \mathrm{~cm}$ in men, $\geq 80 \mathrm{~cm}$ in women), high blood pressure (Systolic $\geq 130$ and/ or diastolic $\geq 85 \mathrm{mmHg}$ ) or drug treatment for hypertension, high LDL-cholesterol $(\geq 130 \mathrm{mg}$ $\mathrm{dL}^{-1}$ ) or drug treatment for high LDL, low HDL cholesterol $\left(<40 \mathrm{mg} \mathrm{dL}^{-1}\right.$ in men and $<50 \mathrm{mg}$ $\mathrm{dL}^{-1}$ in women) or drug treatment for low HDL, high TG $\left(\geq 150 \mathrm{mg} \mathrm{dL}^{-1}\right)$ or drug treatment for high 


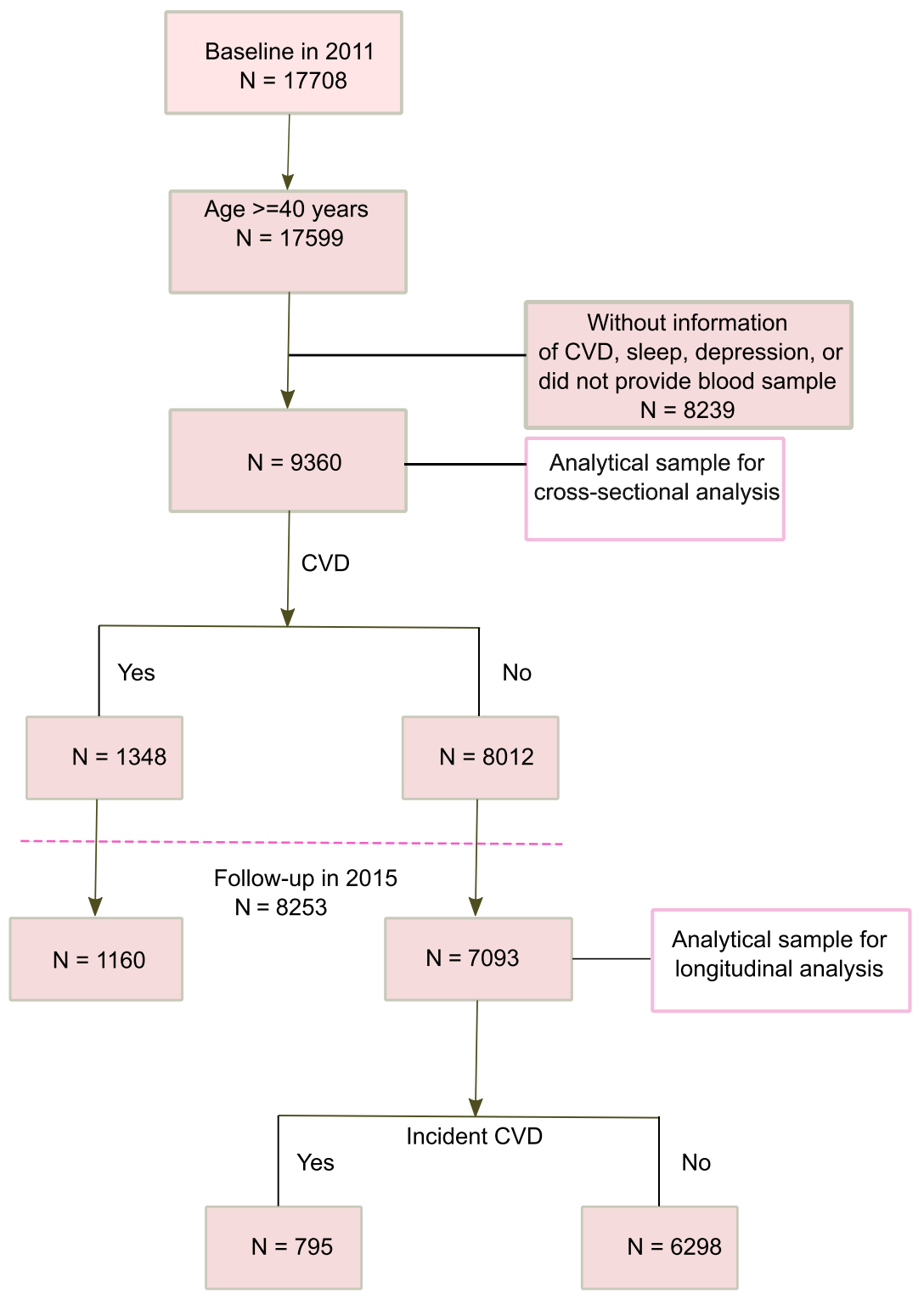

Fig. 1 Sample flow chart.

TG. Lipid-lowering medications were based on selfreport use of western medication for the treatment of abnormal blood lipids.

CircS was based on 6 components including short sleep $\left(<6\right.$ hours day $\left.{ }^{-1}\right)$, depression and four components used to define MetS. A cut-off for CircS was set as $\geq 4$ components (Table 1 ).
Outcome measure: Self-reported CVD

At baseline and follow-up, participants were asked whether they had been diagnosed by a doctor to have stroke or cardiac events (including heart attack, coronary heart disease, angina, congestive heart failure or other heart problems). A positive answer to the question was defined as having CVD. 
Table 1. Definition of metabolic syndrome and circadian syndrome

\begin{tabular}{|c|c|c|c|}
\hline Measure & Categorical cut points & $\begin{array}{l}\text { Included } \\
\text { in MetS }\end{array}$ & $\begin{array}{l}\text { Included } \\
\text { in CircS }\end{array}$ \\
\hline Elevated waist circumference & $\begin{array}{l}\text { Population- and country-specific } \\
\text { definitions ( } \geq 85 \mathrm{~cm} \text { in } \\
\text { men, } \geq 80 \text { in women) }\end{array}$ & $\sqrt{ }$ & $\sqrt{ }$ \\
\hline $\begin{array}{l}\text { Elevated triglycerides } \\
\text { (drug treatment for elevated } \\
\text { triglycerides is an alternate indicator) }\end{array}$ & $\geq 150 \mathrm{mg} \mathrm{dL}^{-1}\left(1.7 \mathrm{mmol} \mathrm{L}^{-1}\right)$ & $\sqrt{ }$ & $\sqrt{ }$ \\
\hline $\begin{array}{l}\text { Reduced HDL-C (drug treatment } \\
\text { for reduced HDL-C } \\
\text { is an alternate indicator) }\end{array}$ & $\begin{array}{l}<40 \mathrm{mg} \mathrm{dL}{ }^{-1}\left(1.0 \mathrm{mmol} \mathrm{L}^{-1}\right) \text { in } \\
\text { men; }<50 \mathrm{mg} \mathrm{dL}^{-1} \\
\left(1.3 \mathrm{mmol} \mathrm{L}^{-1}\right) \text { in women }\end{array}$ & $\sqrt{ }$ & $\sqrt{ }$ \\
\hline $\begin{array}{l}\text { Elevated blood pressure } \\
\text { (antihypertensive drug } \\
\text { treatment in a patient with a history of } \\
\text { hypertension is an alternate indicator) }\end{array}$ & $\begin{array}{l}\text { Systolic } \geq 130 \text { and/or } \\
\text { diastolic } \geq 85 \mathrm{mmHg}\end{array}$ & $\sqrt{ }$ & $\sqrt{ }$ \\
\hline $\begin{array}{l}\text { Elevated fasting glucose (drug treatment of } \\
\text { elevated glucose is an alternate indicator) }\end{array}$ & $\geq 100 \mathrm{mg} \mathrm{dL}^{-1}$ & $\sqrt{ }$ & $\sqrt{ }$ \\
\hline Short sleep & $<6$ hours day ${ }^{-1}$ & & $\sqrt{ }$ \\
\hline Depression symptom & 10 item CES-D score $\geq 10$ & & $\sqrt{ }$ \\
\hline Definition criteria & & $\geq 3$ components & $\geq 4$ components \\
\hline
\end{tabular}

\section{Covariates}

We included the following covariates in the current study: age, gender, education (low, medium, high), residence (urban, rural), physical activity (active, less active, sedentary) [12], smoking (current smokers, ex-smoker or nonsmoker) and alcohol consumption (nondrinker or drinker).

Kidney function was evaluated by the estimated glomerular filtration rate (eGFR) using serum creatinine. The CKD-EPI creatinine equation was used to estimate eGFR [14]. Chronic kidney disease (CKD) was defined as eGFR $<60 \mathrm{~mL} \mathrm{~min}^{-1} / 1.73$ $\mathrm{m}^{2}[15]$.

\section{Statistical analyses}

Multivariable logistic regression analysis was used to examine the associations between MetS or CircS and CVD. The multivariable logistic model adjusted for age, education, smoking, alcohol drinking, physical activity and residence. The predictive power of the two indices (the number of Mets and CircS components were treated as continuous variables) for CVD was assessed using the receiver-operating characteristic (ROC) curve analysis. We used roccomp command in Stata to compare the difference of AUCs between MetS and CircS. To assess the association between MetS/ CircS with incident CVD in 2015, we excluded all those with CVD at baseline in 2011. Genderspecific analyses were conducted. Net reclassification improvement (NRI) was calculated using STATA NRI command [16]. In the NRI calculations, two risk levels were selected; $>15 \%$ and $<15 \%$ CVD. All analyses were conducted using STATA 16.1 (Stata Corporation, College Station, TX, USA). Statistical significance was considered when $P<0.05$ (two sided).

\section{Results}

The mean age of the participants was 59.1 (SD 9.5) years in 2011 (Table 2). The prevalence of CircS and MetS was $39.0 \%$ and $44.7 \%$, respectively. In total, 428 (4.6\%) and 967 (10.3\%) participants had either CircS or MetS alone and 3218 (34.4\%) had both CircS and MetS. The CircS alone group was younger and had the highest prevalence of CKD $(25 \%)$ but lower BMI than other groups. All the participants in the CircS alone group had depression and short sleep duration. Overall, depression 
Table 2. Sample characteristics by MetS and CircS status amongst participants attending China Health and Retirement Longitudinal Study (CHARLS) ( $\mathrm{N}=9360)$

\begin{tabular}{|c|c|c|c|c|c|c|}
\hline & $\begin{array}{l}\text { Total } \\
N=9360\end{array}$ & $\begin{array}{l}\text { Normal } \\
N=4747\end{array}$ & $\begin{array}{l}\text { CircS alone } \\
N=428\end{array}$ & $\begin{array}{l}\text { MetS alone } \\
N=967\end{array}$ & $\begin{array}{l}\text { Both MetS } \\
\text { and CircS } \\
N=3218\end{array}$ & $P$-value \\
\hline Age (years) & $59.1(9.5)$ & $58.6(9.6)$ & $60.7(9.7)$ & $58.2(9.3)$ & $59.8(9.3)$ & $<0.001$ \\
\hline Gender & & & & & & $<0.001$ \\
\hline Male & $4258(45.5 \%)$ & $2678(56.4 \%)$ & 175 (40.9\%) & $456(47.2 \%)$ & 949 (29.5\%) & \\
\hline Female & $5102(54.5 \%)$ & 2069 (43.6\%) & $253(59.1 \%)$ & $511(52.8 \%)$ & 2269 (70.5\%) & \\
\hline Education & & & & & & $<0.001$ \\
\hline Illiterate & $4334(46.3 \%)$ & $2093(44.1 \%)$ & $260(60.7 \%)$ & 369 (38.2\%) & $1612(50.1 \%)$ & \\
\hline Primary school & $2163(23.1 \%)$ & $1169(24.6 \%)$ & $84(19.6 \%)$ & $213(22.0 \%)$ & 697 (21.7\%) & \\
\hline Middle school & 1909 (20.4\%) & $998(21.0 \%)$ & $66(15.4 \%)$ & $249(25.7 \%)$ & $596(18.5 \%)$ & \\
\hline High school or above & $953(10.2 \%)$ & 487 (10.3\%) & $18(4.2 \%)$ & $136(14.1 \%)$ & 312 (9.7\%) & \\
\hline Residence & & & & & & $<0.001$ \\
\hline Rural & $5998(64.1 \%)$ & 3285 (69.2\%) & $320(74.8 \%)$ & 557 (57.6\%) & $1836(57.1 \%)$ & \\
\hline Urban & $3362(35.9 \%)$ & $1462(30.8 \%)$ & $108(25.2 \%)$ & $410(42.4 \%)$ & $1382(42.9 \%)$ & \\
\hline Smoking & & & & & & $<0.001$ \\
\hline Yes & $3610(38.6 \%)$ & $2201(46.4 \%)$ & $167(39.0 \%)$ & $369(38.2 \%)$ & $873(27.1 \%)$ & \\
\hline No & $5749(61.4 \%)$ & 2545 (53.6\%) & $261(61.0 \%)$ & $598(61.8 \%)$ & 2345 (72.9\%) & \\
\hline Drinking & & & & & & $<0.001$ \\
\hline None drinker & $5093(54.4 \%)$ & $2212(46.6 \%)$ & $244(57.0 \%)$ & 508 (52.5\%) & 2129 (66.2\%) & \\
\hline Ex-drinker & $1274(13.6 \%)$ & $712(15.0 \%)$ & $58(13.6 \%)$ & $125(12.9 \%)$ & 379 (11.8\%) & \\
\hline Current drinker & $2993(32.0 \%)$ & $1823(38.4 \%)$ & $126(29.4 \%)$ & $334(34.5 \%)$ & $710(22.1 \%)$ & \\
\hline Physical activity & & & & & & $<0.001$ \\
\hline Active & 2244 (24.0\%) & $1244(26.2 \%)$ & $102(23.8 \%)$ & 257 (26.6\%) & 641 (19.9\%) & \\
\hline Less active & $5741(61.3 \%)$ & 2957 (62.3\%) & $262(61.2 \%)$ & 567 (58.6\%) & 1955 (60.8\%) & \\
\hline Sedentary & $1375(14.7 \%)$ & $546(11.5 \%)$ & $64(15.0 \%)$ & $143(14.8 \%)$ & 622 (19.3\%) & \\
\hline BMI $\left(\mathrm{kg} \mathrm{m}^{-2}\right)$ & $23.6(3.9)$ & $22.1(3.3)$ & $22.5(3.5)$ & $24.9(3.6)$ & $25.7(3.8)$ & $<0.001$ \\
\hline Systolic BP & $131(22)$ & $124(20)$ & $132(22)$ & $135(21)$ & $139(22)$ & $<0.001$ \\
\hline Diastolic BP & 76 (12) & $738(11)$ & 76 (12) & 79 (12) & $80(12)$ & $<0.001$ \\
\hline $\begin{array}{l}\text { High-sensitivity } \\
\text { CRP }\left(\mathrm{mg} \mathrm{dL}^{-1}\right)\end{array}$ & $1.06(.56-2.2)$ & $.82(.47-1.74)$ & $.91(.52-2.165)$ & $1.18(.63-2.31)$ & $1.41(.75-2.83)$ & $<0.001$ \\
\hline CVD in 2011 & $1348(14.4 \%)$ & 445 (9.4\%) & 80 (18.7\%) & 98 (10.1\%) & 725 (22.5\%) & $<0.001$ \\
\hline Hypertension & 3749 (41.0\%) & $1108(23.5 \%)$ & $189(45.1 \%)$ & 455 (48.5\%) & 1997 (65.4\%) & $<0.001$ \\
\hline CKD & 1054 (14.9\%) & $480(13.5 \%)$ & $83(25.3 \%)$ & 87 (12.2\%) & 404 (16.5\%) & $<0.001$ \\
\hline Anaemia & $1188(13.0 \%)$ & $698(15.1 \%)$ & 67 (15.9\%) & 104 (10.9\%) & 319 (10.3\%) & $<0.001$ \\
\hline Central obesity & 4899 (52.3\%) & $1227(25.8 \%)$ & $198(46.3 \%)$ & 707 (73.1\%) & 2767 (86.0\%) & $<0.001$ \\
\hline Heart Problems & $1188(12.7 \%)$ & 393 (8.3\%) & $68(15.9 \%)$ & 88 (9.1\%) & 639 (19.9\%) & $<0.001$ \\
\hline Stroke & 215 (2.3\%) & 61 (1.3\%) & $14(3.3 \%)$ & $13(1.3 \%)$ & 127 (4.0\%) & $<0.001$ \\
\hline $\begin{array}{l}\text { Elevated } \\
\text { plasma glucose }\end{array}$ & $5536(59.1 \%)$ & $1862(39.2 \%)$ & 249 (58.2\%) & 705 (72.9\%) & $2720(84.5 \%)$ & $<0.001$ \\
\hline $\begin{array}{l}\text { Elevated serum } \\
\text { triglycerides }\end{array}$ & $2804(30.0 \%)$ & $264(5.6 \%)$ & $44(10.3 \%)$ & 314 (32.5\%) & 2182 (67.8\%) & $<0.001$ \\
\hline
\end{tabular}


Table 2 (Continued)

\begin{tabular}{|c|c|c|c|c|c|c|}
\hline & $\begin{array}{l}\text { Total } \\
N=9360\end{array}$ & $\begin{array}{l}\text { Normal } \\
N=4747\end{array}$ & $\begin{array}{l}\text { CircS alone } \\
N=428\end{array}$ & $\begin{array}{l}\text { MetS alone } \\
N=967\end{array}$ & $\begin{array}{l}\text { Both MetS } \\
\text { and CircS } \\
N=3218\end{array}$ & $P$-value \\
\hline Elevated blood pressure & $5117(54.7 \%)$ & $1619(34.1 \%)$ & $251(58.6 \%)$ & 661 (68.4\%) & $2586(80.4 \%)$ & $<0.001$ \\
\hline $\begin{array}{l}\text { Reduced serum } \\
\text { HDL-C }\end{array}$ & $3942(42.1 \%)$ & $695(14.6 \%)$ & $114(26.6 \%)$ & $514(53.2 \%)$ & $2619(81.4 \%)$ & $<0.001$ \\
\hline Depression & $3596(38.4 \%)$ & $1574(33.2 \%)$ & $428(100.0 \%)$ & $0(0.0 \%)$ & $1594(49.5 \%)$ & $<0.001$ \\
\hline Short sleep & $2822(30.1 \%)$ & $1150(24.2 \%)$ & $428(100.0 \%)$ & $0(0.0 \%)$ & $1244(38.7 \%)$ & $<0.001$ \\
\hline Circadian syndrome & $3646(39.0 \%)$ & $0(0.0 \%)$ & $428(100.0 \%)$ & $0(0.0 \%)$ & $3218(100.0 \%)$ & $<0.001$ \\
\hline Metabolic syndrome & $4185(44.7 \%)$ & $0(0.0 \%)$ & $0(0.0 \%)$ & 967 (100.0\%) & $3218(100.0 \%)$ & $<0.001$ \\
\hline CVD in 2015 & $1954(23.7 \%)$ & $726(17.1 \%)$ & $111(30.2 \%)$ & $172(20.2 \%)$ & 945 (33.9\%) & $<0.001$ \\
\hline
\end{tabular}

Data are presented as mean (SD) or median (IQR) for continuous measures, and $\mathrm{n}(\%)$ for categorical measures.

Table 3. Association (odds ratio; 95\% confidence interval, CI) between metabolic syndrome (MetS) and circadian syndrome (CircS) and CVD amongst adults attending China Health and Retirement Longitudinal Study (CHARLS)

\begin{tabular}{|c|c|c|c|c|}
\hline & \multicolumn{2}{|l|}{ MetS } & \multicolumn{2}{|l|}{ CircS } \\
\hline & $\begin{array}{l}\text { Per one of MetS } \\
\text { components (continuous) }\end{array}$ & MetS (yes vs no) & $\begin{array}{l}\text { Per one of CircS } \\
\text { components (continuous) }\end{array}$ & CircS (yes vs no) \\
\hline \multicolumn{5}{|c|}{ Prevalent CVD in 2011} \\
\hline \multicolumn{5}{|c|}{ Men $(n=4258)$} \\
\hline Model 1 & $1.45(1.36-1.55)$ & $2.42(2.01-2.93)$ & $1.47(1.38-1.57)$ & $2.87(2.37-3.48)$ \\
\hline Model 2 & $1.40(1.31-1.50)$ & $2.21(1.82-2.69)$ & $1.45(1.36-1.54)$ & $2.69(2.21-3.27)$ \\
\hline \multicolumn{5}{|c|}{ Women $(n=5102)$} \\
\hline Model 1 & $1.33(1.26-1.41)$ & $1.84(1.57-2.16)$ & $1.39(1.32-1.46)$ & $2.34(2.00-2.74)$ \\
\hline Model 2 & $1.30(1.23-1.37)$ & $1.71(1.46-2.01)$ & $1.38(1.31-1.45)$ & $2.23(1.90-2.62)$ \\
\hline \multicolumn{5}{|c|}{ Incident CVD in 2015} \\
\hline \multicolumn{5}{|c|}{$\operatorname{Men}(n=3293)$} \\
\hline Model 1 & $1.22(1.12-1.32)$ & $1.57(1.24-1.98)$ & $1.24(1.15-1.34)$ & $1.67(1.31-2.14)$ \\
\hline Model 2 & $1.21(1.11-1.31)$ & $1.53(1.21-1.94)$ & $1.24(1.15-1.34)$ & $1.66(1.30-2.13)$ \\
\hline \multicolumn{5}{|c|}{ Women $(n=3800)$} \\
\hline Model 1 & $1.19(1.11-1.28)$ & $1.57(1.28-1.92)$ & $1.23(1.15-1.31)$ & $1.73(1.42-2.12)$ \\
\hline Model 2 & $1.19(1.11-1.28)$ & $1.54(1.26-1.90)$ & $1.23(1.15-1.31)$ & $1.72(1.41-2.11)$ \\
\hline
\end{tabular}

Values are odds ratio (95\%CI) derived from multivariable logistic regression models.

Model 1 adjusted for age.

Model 2 further adjusted for education, smoking, alcohol drinking, physical activity and residence (urban/rural).

was more common than short sleep amongst those with CircS (55.5\% vs $45.9 \%)$.

Both MetS and CircS were directly associated with prevalent CVD. The prevalence of CVD at the baseline was $22.1 \%$ amongst those with CircS and $19.7 \%$ in those with MetS. The odds ratios for prevalent CVD in fully adjusted multivariable analysis (Model 2) were 2.69 (95\%CI 2.21-3.27) and $2.21(1.82-2.69)$ in men for the CircS and MetS, respectively (Table 3), and 2.23 (1.90-2.62) and $1.71(1.46-2.01)$, respectively, in women. The five-year incidence of CVD was $15.1 \%$ in $\mathrm{CircS}$ and $14.0 \%$ in MetS. The odds ratios for incident CVD 
Table 4. Association between metabolic syndrome (MetS) and circadian syndrome (CircS) status and CVD amongst adults attending China Health and Retirement Longitudinal Study (CHARLS)

\begin{tabular}{|c|c|c|c|c|}
\hline & \multicolumn{4}{|c|}{ Health status } \\
\hline & Normal & CircS alone & Mets alone & CircS and Mets \\
\hline \multicolumn{5}{|c|}{ Prevalent CVD in 2011} \\
\hline \multicolumn{5}{|c|}{ Men $(n=4258)$} \\
\hline Model 1 & 1.00 & $1.88(1.22-2.90)$ & $1.32(0.94-1.83)$ & $3.25(2.64-4.01)$ \\
\hline Model 2 & 1.00 & $1.98(1.28-3.06)$ & $1.22(0.87-1.71)$ & $2.95(2.38-3.65)$ \\
\hline \multicolumn{5}{|c|}{ Women $(n=5102)$} \\
\hline Model 1 & 1.00 & $2.07(1.48-2.90)$ & $0.92(0.66-1.27)$ & $2.34(1.97-2.78)$ \\
\hline Model 2 & 1.00 & $2.13(1.52-3.00)$ & $0.86(0.62-1.20)$ & $2.18(1.83-2.60)$ \\
\hline \multicolumn{5}{|c|}{ Incident CVD in 2011-2015 } \\
\hline \multicolumn{5}{|c|}{ Men $(n=3293)$} \\
\hline Model 1 & 1.00 & $1.77(1.20-2.60)$ & $1.34(1.02-1.75)$ & $2.71(2.25-3.27)$ \\
\hline Model 2 & 1.00 & $1.87(1.26-2.76)$ & $1.27(0.97-1.67)$ & $2.55(2.11-3.09)$ \\
\hline \multicolumn{5}{|c|}{ Women $(n=3800)$} \\
\hline Model 1 & 1.00 & $2.05(1.50-2.79)$ & $1.12(0.87-1.46)$ & $2.20(1.89-2.57)$ \\
\hline Model 2 & 1.00 & $2.10(1.54-2.87)$ & $1.06(0.82-1.38)$ & $2.11(1.81-2.46)$ \\
\hline
\end{tabular}

Values are odds ratio (95\%CI) derived from multivariable logistic regression models.

Model 1 adjusted for age.

Model 2 further adjusted for education, smoking, alcohol drinking, physical activity and residence (urban/rural).

were $1.66(1.30-2.13)$ and $1.53(1.21-1.94)$ in men; $1.72(1.41-2.11)$ and $1.54(1.26-1.90)$ in women for CircS and MetS, respectively.

Having both MetS and CircS was associated with more than twice the risk of developing CVD in both men and women. CircS only was associated with $87 \%$ and $110 \%$ increased risk of developing CVD in men and women, respectively. MetS by itself was not statistically associated with CVD (Table 4).

The number of CircS components had a better predictive power for both prevalent and incident CVD than the number of MetS components as indicated by the area under the ROC (AUC) (Fig. 2). AUC values for CVD in 2011 were higher for CircS than MetS in both men $(0.659$ 95\%CI (0.634$0.684)$ vs 0.635 (95\%CI (0.610-0.661)) and women $(0.652$ (95\%CI $0.632-0.672)$ vs 0.619 (95\%CI 0.599-0.640)). CircS had a higher specificity but a lower sensitivity for CVD than MetS (Table S1). Both MetS and CircS had a higher sensitivity for CVD in men than in women.

Net reclassification improvement after adding the risk score (short sleep and depression) to components of MetS for improved risk classification (CVD risk above $15 \%$ or below $15 \%$ ) was 0.040 for prevalent CVD in $2011(P=0.003)$ and 0.038 for incident CVD in 2011-2015 ( $P=0.035)$.

\section{Discussion}

In this large population-based study of Chinese adults, we found that the CircS is a better predictor for both prevalent and incident CVD than the MetS. Having both MetS and CircS was associated with a doubled risk of CVD compared with people without any of these syndromes. Having CircS alone without MetS was associated with prevalent and incident CVD in both men and women, but in participants with MetS alone no statistically significant association with prevalent CVD was observed. Assessing the effect of the exposure on the incidence provides information of the time sequence that the exposure preceded the outcome, whilst the cross-sectional analysis at baseline cannot do it.

In our study, the MetS was associated with a doubled risk of CVD. The finding is in line with previous studies. In a meta-analysis of 87 studies, MetS had an RR of 2.35 (95\%CI 2.02-2.73) for CVD [5]. To the best of our knowledge, no study has assessed the prevalence of CircS and the 

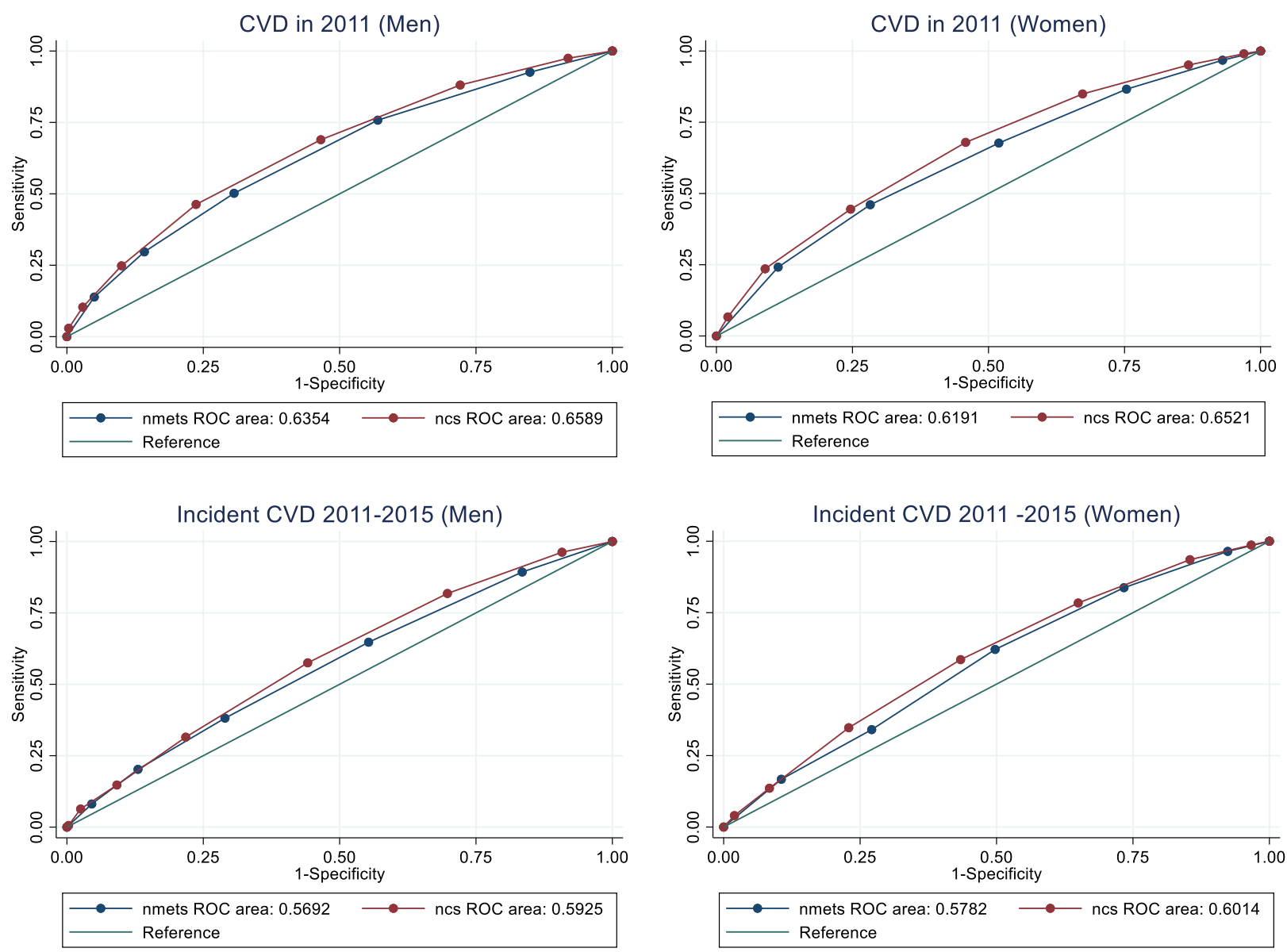

Fig. 2 ROC for prevalent and incident CVD based on number of components of metabolic syndrome and circadian syndrome. There was a significant difference in AUC between the number of metabolic syndrome components and number of circadian syndrome components in both genders. nmets: number of metabolic syndrome components. ncs: number of circadian syndrome components.

association between CircS and CVD. Two additional components, short sleep and depression, were added into the components of MetS to construct the CircS. Existing evidence suggests that both short sleep and depression are associated with increased risk of CVD [17, 18]. As expected, CircS was associated with CVD in our study. In fact, CircS has a better predictive value than MetS as indicated by AUC.

The interesting finding of this study is that MetS alone was not associated with the risk of CVD, but CircS alone was. Our findings emphasize the rationale and importance of incorporating short sleep and depression into the MetS risk factor cluster and construct the CircS. The prevalence of CircS alone was $4.6 \%$. If we only use MetS to prevent and predict CVD, this group will be missed. One of the important clinical features of this group is the high prevalence of CKD. Around 25\% of the participants in this group had CKD. The significant association between CircS and CVD in this group may be partly due to CKD, but further adjusting for CKD did not attenuate the association between CircS and CVD. Daily rhythmicity is required for proper kidney function, which is the site of one of the most active peripheral clocks. Disrupted circadian rhythms have been associated with the development of CKD in both humans and animal models [19]. Therefore, it is not surprising that there was a significant association between CircS and CKD in our study and strengthens the hypothesis that disturbance of circadian rhythms is an important underlying etiological factor. 
CircS alone group had lower BMI than other groups. High BMI is associated with increased risk of MetS, but not all overweight or obese people have MetS, and vice versa [20]. It is possible that in this group, circadian disruption is an even more central underlying etiological factor.

The rationale for the concept of CircS has been provided in its initial publication [10]. There is a strong link between circadian rhythms and MetS [21]. We found that CircS is a better substitute for the MetS. Similarly, all of the components of CircS have been shown to be related to circadian disruption [10]. Thus, circadian disruption could underline the common aetiology of MetS and CircS. Several factors have been proposed to be responsible for the disruption of circadian rhythm in our modern life, including the extensive use of artificial light, controlled ambient temperature and constant food availability, shift work, jet travel with different time zones [10]. All these factors have been shown to be related to the components of CircS used in the current study as well as CVD. For example, findings from a review of 85 studies suggest that artificial light at night had negative effects on psychological, cardiovascular and metabolic functions [22]. Mounting evidence suggests that sleep deprivation has metabolic consequences and increases the risk of type 2 diabetes and/or obesity [23]. Furthermore, a high fat diet, common in modern society, affects sleep quality [24]. In a clinical laboratory, Sheer et al found there is a causal link between circadian misalignment and metabolic homeostasis [25].

The limitation of this study is the short follow-up duration. We only have self-reported sleep duration rather than objectively measured sleep parameters. The original proposed CircS includes nonalcoholic fatty liver disease (NAFLD). It is known that the prevalence of NAFLD is high in China ( 20\%) [26]. However, we do not have information on NAFLD. The use of self-reported CVD is another limitation. However, self-reported CVD has been shown to be reliable to ascertain nonfatal events $[27,28]$, but fatal events remain undetected. The strength of the study is the large sample size and representative of the general population. This is the first study to assess the association between CircS and CVD risk. More studies are needed to validate our findings in other populations, and using more objective measurements of disturbances of the circadian rhythm. As the assessment of short sleep and depression is relatively easy and cheap, they should be routinely measured in combination of MetS in clinical settings to prevent CVD.

In conclusion, both MetS and CircS can predict CVD in the Chinese population and the use of CircS can predict CVD better. Adding sleep quality and depression to the MetS construct to create the CircS might be both a cost- and health effective approach to prevention of CVD.

\section{Acknowledgements}

We would like to acknowledge the China Health and Retirement Longitudinal Study (CHARLS) team for providing data and the training of using the data set. ZS, JT, NK, GA, SEO, CB, NS and PZ contributed to the design and conduct of the study and the interpretation of the data. ZS and PZ contributed to the analysis and interpretation of data. ZS wrote the first draft of the manuscript, and all authors contributed to subsequent drafts and approved the final version for submission. ZS has full access to all the data in the study and takes responsibility for the integrity of the data and the accuracy of the data analysis.

\section{Sources of Funding}

Open Access funding was provided by the Qatar National Library.

\section{Conflict of interest}

All the authors report no conflicts.

\section{Author contribution}

Zumin Shi: Conceptualization (equal); Data curation (lead); Formal analysis (lead); Methodology (equal); Project administration (lead); Resources (equal); Software (equal); Visualization (equal); Writing-original draft (lead); Writing-review \& editing (equal). Jaakko Tuomilehto: Methodology (equal); Writing-review \& editing (equal). Noga Kronfeld-Schor: Conceptualization (equal); Writing-review \& editing (equal). George Alberti: Writing-review \& editing (equal). Naftali Stern: Writing-review \& editing (equal). Sam E1-Osta: Writing-review \& editing (equal). Carmel Bilu: Writing-review \& editing (equal). Haim Einat: Writing-review \& editing (equal). Paul Zimmet: Conceptualization (equal); Investigation (equal); Methodology (equal); Project administration 
(equal); Supervision (lead); Writing-review \& editing (equal).

\section{Data and resource availability}

The data are available from the CHARLS website.

\section{References}

1 Roth GA, Johnson C, Abajobir A et al. Global, regional, and national burden of cardiovascular diseases for 10 causes, 1990 to 2015. J Am Coll Cardiol 2017; 70: 1-25.

2 Yang ZJ, Liu J, Ge JP et al. Prevalence of cardiovascular disease risk factor in the Chinese population: the 2007-2008 China National Diabetes and Metabolic Disorders Study. Eur Heart J 2012; 33: 213-20.

3 Alberti KG, Eckel RH, Grundy SM et al. Harmonizing the metabolic syndrome: a joint interim statement of the International Diabetes Federation Task Force on Epidemiology and Prevention; National Heart, Lung, and Blood Institute; American Heart Association; World Heart Federation; International Atherosclerosis Society; and International Association for the Study of Obesity. Circulation 2009; 120: 1640-5.

4 Lakka HM, Laaksonen DE, Lakka TA et al. The metabolic syndrome and total and cardiovascular disease mortality in middle-aged men. JAMA 2002; 288: 2709-16.

5 Mottillo $\mathrm{S}$, Filion KB, Genest $\mathrm{J}$ et al. The metabolic syndrome and cardiovascular risk a systematic review and meta-analysis. J Am Coll Cardiol 2010; 56: 1113-32.

$6 \mathrm{Lu} \mathrm{J}$, Wang L, Li M et al. Metabolic syndrome among adults in China: The 2010 China noncommunicable disease surveillance. J Clin Endocrinol Metabol 2016; 102: 507-15.

7 Reaven GM. Banting lecture 1988. Role of insulin resistance in human disease. Diabetes 1988; 1988(37): 1595-607.

8 Alberti KG, Zimmet P, Shaw J, Group IDFETFC. The metabolic syndrome-a new worldwide definition. Lancet 2005; 366: 1059-62.

9 Groop L. Genetics of the metabolic syndrome. Br J Nutr 2000; 83(Supp1 1): S39-48.

10 Zimmet P, Alberti K, Stern N et al. The Circadian Syndrome: is the Metabolic Syndrome and much more!. J Intern Med 2019; 286: 181-91.

11 Zhao Y, Hu Y, Smith JP, Strauss J, Yang G. Cohort profile: the China Health and Retirement Longitudinal Study (CHARLS). Int $J$ Epidemiol 2014; 43: 61-8.

12 Wang S, Chen R, Liu Q, Shu Z, Zhan S, Li L. Prevalence, awareness and treatment of chronic kidney disease among middle-aged and elderly: The China Health and Retirement Longitudinal Study. Nephrology 2015; 20: 474-84.

13 Andresen EM, Malmgren JA, Carter WB, Patrick DL. Screening for depression in well older adults: evaluation of a short form of the CES-D (Center for Epidemiologic Studies Depression Scale). Am J Prev Med 1994; 10: 77-84.

14 Inker LA, Schmid CH, Tighiouart $\mathrm{H}$ et al. Estimating glomerular filtration rate from serum creatinine and cystatin C. $N$ Engl J Med 2012; 367: 20-9.

15 Webster AC, Nagler EV, Morton RL, Masson P. Chronic kidney disease. Lancet 2017; 389: 1238-52.
16 Pencina MJ, D'Agostino RB Sr, D’Agostino RB Jr, Vasan RS. Evaluating the added predictive ability of a new marker: from area under the ROC curve to reclassification and beyond. Stat Med 2008; 27: 157-72; discussion 207-12.

17 Hall MH, Brindle RC, Buysse DJ. Sleep and cardiovascular disease: Emerging opportunities for psychology. Am Psychol 2018; 73: 994-1006.

18 Hare DL, Toukhsati SR, Johansson P, Jaarsma T. Depression and cardiovascular disease: a clinical review. Eur Heart $J$ 2014; 35: 1365-72.

19 Carriazo S, Ramos AM, Sanz AB et al. A poorly recognized feature of CKD. Toxins (Basel) 2020; 12.

20 Bays HE, Chapman RH, Grandy S, Group SI. The relationship of body mass index to diabetes mellitus, hypertension and dyslipidaemia: comparison of data from two national surveys. Int $J$ Clin Pract 2007; 61: 737-47.

21 Maury E, Ramsey KM, Bass J. Circadian rhythms and metabolic syndrome: from experimental genetics to human disease. Circ Res 2010; 106: 447-62.

22 Cho Y, Ryu SH, Lee BR, Kim KH, Lee E, Choi J. Effects of artificial light at night on human health: A literature review of observational and experimental studies applied to exposure assessment. Chronobiol Int 2015; 32: 1294-310.

23 Knutson KL, Spiegel K, Penev P, Van Cauter E. The metabolic consequences of sleep deprivation. Sleep Med Rev 2007; 11: 163-78.

24 Cao Y, Wittert G, Taylor AW, Adams R, Shi Z. Associations between macronutrient intake and obstructive sleep Apnoea as well as self-reported sleep symptoms: results from a cohort of community dwelling Australian men. Nutrients 2016; 8: 207.

25 Scheer FA, Hilton MF, Mantzoros CS, Shea SA. Adverse metabolic and cardiovascular consequences of circadian misalignment. Proc Natl Acad Sci U S A 2009; 106: 4453-8.

26 Li Z, Xue J, Chen P, Chen L, Yan S, Liu L. Prevalence of nonalcoholic fatty liver disease in mainland of China: a metaanalysis of published studies. J Gastroenterol Hepatol 2014; 29: $42-51$.

27 Barr EL, Tonkin AM, Welborn TA, Shaw JE. Validity of selfreported cardiovascular disease events in comparison to medical record adjudication and a statewide hospital morbidity database: the AusDiab study. Intern Med J 2009; 39: 49-53.

28 Engstad T, Bonaa KH, Viitanen M. Validity of self-reported stroke: The Tromso Study. Stroke 2000; 31: 1602-7.

Correspondence: Zumin Shi, Human Nutrition Department, College of Health Sciences, QU Health, Qatar University, P.O. Box 2713 Doha, Qatar.

(fax: +974 4403 4801; e-mail: zumin@qu.edu.qa).

\section{Supporting Information}

Additional Supporting Information may be found in the online version of this article:

Table S1. Sensitivity, specificity and AUC by metabolic syndrome and circadian syndrome in predicting CVD risk among adults in China. 


\section{University Library}

\section{- M M N E R VA A gateway to Melbourne's research publications}

Minerva Access is the Institutional Repository of The University of Melbourne

Author/s:

Shi, Z;Tuomilehto, J;Kronfeld-Schor, N;Alberti, GK;Stern, N;El-Osta, A;Bilu, C;Einat, H;Zimmet, P

Title:

The circadian syndrome predicts cardiovascular disease better than metabolic syndrome in Chinese adults.

Date:

2021-06

Citation:

Shi, Z., Tuomilehto, J., Kronfeld-Schor, N., Alberti, G. K., Stern, N., El-Osta, A., Bilu, C., Einat, H. \& Zimmet, P. (2021). The circadian syndrome predicts cardiovascular disease better than metabolic syndrome in Chinese adults.. J Intern Med, 289 (6), pp.851-860. https:// doi.org/10.1111/joim.13204.

Persistent Link:

http://hdl.handle.net/11343/274136

License:

CC BY 